carcinoma lung. CT scan and MRI may help the diagnosis, but a positive cytological examination of the cerebrospinal fluid is confirmatory. Intrathecal chemotherapy with involved field radiotherapy is the mainstay of treatment.

A 60-year-old female presented with left lower lobe lung nodule of a poorly differentiated adenocarcinoma. CT Thorax showed a mass in superior segment of $(L)$ lower lobe involving segmental bronchus and another metastatic nodular lesion in the (L) paraortic region with multiple nodules scattered throughout the $(L)$ lung parenchyma. Following six cycles of Gemcitabine and Carboplatin, a repeat $\mathrm{CT}$ scan after six months revealed significant resolution in size of the lesions. L eft lower lobectomy with lymph node dissection was performed. $\mathrm{H}$ istopathology revealed residual Adenocarcinoma with invasion into visceral pleura with vascular invasion and 3 nodes showing metastasis. Three more cycles of chemotherapy with paclitaxel and carboplatin followed.

Patient first complained of headache four months later but CT brain was normal. She was found to have hypertension and treatment was started for the same. She remained asymptomatic for two months when she presented with headache, vomiting and altered sensorium. CT and MRI Brain were normal. PET scan was normal. CSF Cytology clinched the diagnosis [Figures 1 and 2].

Intrathecal Chemotherapy with M ethotrexate and Cytarabine showed some improvement. Patient expired two weeks later.

M eningeal carcinomatosis is a diffuse infiltration of leptomeninges and sub arachnoid spaces by malignant cells metastasizing from systemic cancer. It has a pleomorphic clinical presentation affecting cerebrum, cranial nerves or spinal cord. The probability of

\title{
Meningeal carcinomatosis from pulmonary adenocarcinoma
}

Dear Sir,

Meningeal carcinomatosis without brain parenchymal involvement is a rare metastatic manifestation of

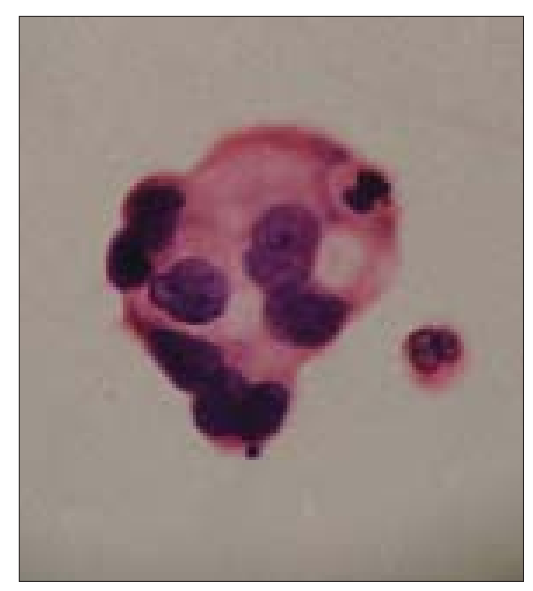

Figure 1: Single cancer cell on CSF picture 


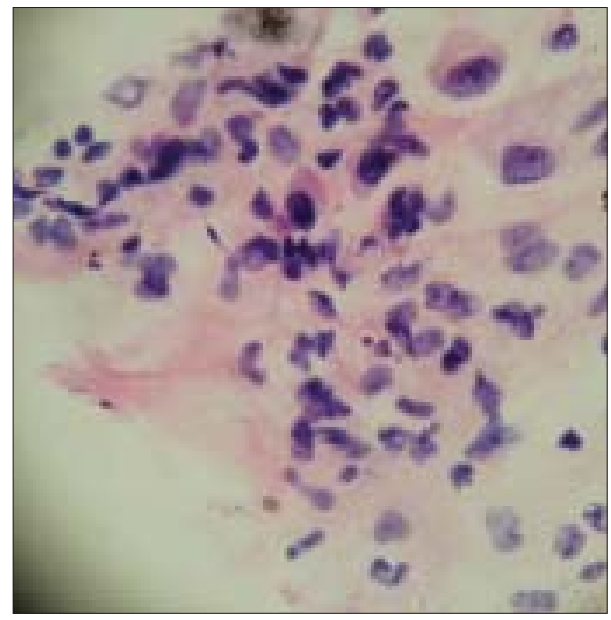

Figure 2: Group of malignant cells on CSF picture

developing meningeal carcinomatosis was $2.7 \%$ at three years and $7.8 \%$ at five years. ${ }^{[1]}$ It spreads mainly by hematogenous route and CSF is diagnostic. $30-60 \%$ have co-existent parenchymal brain metastasis. Treatment is I ntrathecal Chemotherapy -M ethotrexate, Cytarabine, Thiotepa. ${ }^{[2]}$ Intrathecal Gemcitabine has been used in non small cell lung cancer. ${ }^{[3]}$ Intravenous teniposide may be used along with intrathecal chemotherapy. ${ }^{[4]}$ Involved field radiotherapy is reserved for bulky metastatic disease.

The presentation of meningeal carcinomatosis without systemic metastases is rare. Our patient had a metastatic lung nodule to begin with but the disease was well controlled at the time of presentation with meningeal carcinomatosis. The symptoms of headache, vomiting with raised blood pressure along with normal imaging led to a delay in doing CSF cytology. Meningeal carcinomatosis may prove difficult to diagnose as imaging may be misleading. This warrants a high index of suspicion and early CSF cytology.

Doctor AM, Sharma S1, Maheshwari M Oncosurgery Department, Bombay Hospital Medical Research Centre, MRC, Marine Lines, Mumbai - 400 020, India. 'Mumbai Cancer Clinic, Sagar Fortune, Waterfield Road, Bandra (West), Mumbai - 400 050, India.

Correspondence to: Dr. Azmat M Doctor, E-mail: azmatdoc@hotmail.com

\section{References}

1. Ohnoshi T, Ueoka H, Kiura K, Mima Y, Miyatake K, Genba K, et al. Meningeal carcinomatosis in patients with small cell lung cancer. Jpn J Thorac Dis 1993;31:324-9.

2. Chamberlain MC, Kormanik PA. Complications associated with intraventrcular chemotherapy in patients with leptomeningeal metatases. J Neurosurg 1997;87:694-9.
3. Chen YM, Chen MC, Tsai CM, Perng RP. Intrathecal Gemctabine chemotherapy for non small cell lung cancer in patients with Meningeal carcinomatosis. Lung Cancer 2003;40:99-101.

4. van der Graaf WT, Haaxma-Reiche H, Burghouts JT, Postmus PE. Teniposide for meningeal carcinomatosis of small cell lung cancer. Lung Cancer 1993; 10:247-9. 